\title{
Le théâtre des vanités mondaines : le Bois de Boulogne dans les chroniques zoliennes
}

Journaliste collaborant à plusieurs périodiques en France et à l'étranger parallèlement à son activité de romancier, de nouvelliste, de dramaturge, de critique littéraire et artistique, Émile Zola est un chroniqueur parisien des pieds à la tête ${ }^{1}$. Si, parmi ses œuvres de fiction, il n'y en a qu'un certain nombre pour lesquelles la capitale française constitue le lieu d'action - la moitié des Rougon-Macquart et un tiers des Trois villes se passent à Paris -, le volet journalistique de l'ouvre zolienne est entièrement lié à la réalité parisienne qu'il décrit et analyse, qu'il pastiche souvent, et qui lui sert de source inépuisable d'inspiration. En même temps portraitiste de l'espace de la ville, connaisseur de ses habitants et chroniqueur de sa vie quotidienne, Zola est en effet un " authentique fabricant d' "articles de Paris" " (Dezalay, 1967, p. 118).

À côté des centaines de textes consacrés aux sujets "sérieux " (réalité urbaine, politique, sociologie, etc.), de nombreuses chroniques sont dédiées au Paris mondain, " ses mœurs, ses rituels, ses lieux à la mode, ses divertissements » (Mitterand, 2013, p. 65). L'auteur ne s'y montre guère admiratif devant le mode de vie adopté par les élites du Second Empire ; à travers sa critique, souvent très virulente, se lit non seulement la haine et le mépris qu'il vouait pour le régime, mais aussi son indignation personnelle face à toutes les transformations, aussi bien physiques que sociologiques, que Paris a subies sous le règne de Napoléon III.

Anna Kaczmarek-Wiśniewska - docteur ès lettres habilitée à diriger des recherches, maitre de conférences à l'Institut de Culture et de Langue françaises de l'Université d'Opole. Adresse pour correspondance : Université d'Opole, Institut de Culture et de Langue françaises, pl. Kopernika 11, 45- 287 Opole, Pologne ; e-mail : akaczmarek@uni.opole.pl

1. La présente esquisse prend pour base notre ouvrage consacré aux chroniques journalistiques de Zola : Kaczmarek-Wiśniewska, A. (2017). La vie quotidienne à Paris suivant les chroniques d'Émile Zola: un regard oblique. Opole, Pologne: Wydawnictwo Uniwersytetu Opolskiego. 
Ce nouveau Paris, agrandi et entièrement réaménagé, est la ville des rêves de l'empereur. Après les années passées en exil à Londres, " modèle d'urbanisme et d'hygiène " (De Moncan, 2012, p. 27) pour l'Europe de l'époque, Louis-Napoléon Bonaparte considérait sa capitale comme une cité ayant un retard énorme par rapport à d'autres grandes villes européennes; c'est pourquoi il « s'est senti contraint de remodeler Paris justement pour lui donner l'aspect et l'allure correspondant à la réputation que la ville avait dans le monde " (Cancellieri, 2013, p. 163). Il fallait dire adieu au vieux Paris sombre, puant et sordide, celui des Mystères de Paris d'Eugène Sue. Les chiffres témoignent de l'importance de l'entreprise : « En seulement dix-sept ans, 20000 immeubles sont abattus et remplacés par 30000 nouveaux, 300 kilomètres de voies sont tracés et illuminés, 600 kilomètres d'égouts construits et un million d'arbres plantés» (Cancellieri, 2013, p. 82). Henri Mitterand décrit les résultats de la transformation de la capitale comme suit :

Jamais le paysage de Paris n’a été si vite ni si complètement remodelé [...]. Cette ville modernisée, avec ses nouvelles voies droites et larges, qui sont passées sur le ventre de milliers de vieilles maisons, avec aussi ses bâtisses neuves, hautes et cossues, ses places en croix ou en étoile, s'ouvre à des milliers de provinciaux immigrants et de voyageurs étrangers, curieux des larges perspectives, des expositions universelles (1855 et 1867), des défilés militaires, des cérémonies et des fêtes. Il fallait pour les accueillir des gares, des marchés, des magasins, des théâtres et aussi des hôpitaux et des bibliothèques. D’où le surgissement d'un nouveau Paris monumental [...]. (Mitterand, 2008, p. 32-33)

Selon le Dictionnaire d'Émile Zola, le baron Georges-Eugène Haussmann, l'architecte de la métamorphose de Paris, "un des grands constructeurs du siècle, [...] qui [a] marqué [son] époque par l'ampleur de [son] œuvre », est sans doute " un de ces hommes que Zola admire » (Becker, Gourdin-Servenière et Lavielle, 1993, p. 180). On pourrait donc s'attendre à ce que, pour un Zola fasciné par le développement et la modernisation de la capitale, tout le Paris "post-haussmanien ", la somptuosité des nouvelles constructions, le charme des vieux bâtiments rénovés, des larges boulevards, des parcs et des jardins récemment aménagés, ainsi que celui de la société élégante qui l'habite, soit beaucoup plus captivant que les recoins du Paris médiéval ou le paysage de la campagne entourant la Ville Lumière qu'il aime à dépeindre dans ses chroniques. Or, il n'en est rien ; bien au contraire : dans l'œuvre de Zola, le remodelage haussmannien de la capitale est évoqué de manière très nuancée : certaines réalisations sont minutieusement décrites, d'autres passées sous silence. En effet, l'auteur voit dans les travaux d'Haussmann avant tout une dispendieuse politique du prestige que le régime grotesque de Napoléon III, usurpateur et hypocrite, s'efforce de se bâtir. Ainsi, la ville transfigurée avec ses nouvelles upper classes semble le décevoir profondément, et la vie des habitants des beaux quartiers ne provoque chez lui qu'un sourire ironique et des paroles acerbes. 
C’est que l'écrivain-journaliste s'avère particulièrement sensible à un aspect particulier du nouveau Paris, à savoir la démesure, l'ostentation visible dans l'étalage des signes extérieurs de la richesse des élites. En effet, comme l’observe Henri Mitterand, Paris compte à l'époque "plus de 2000 millionnaires [...] parmi lesquels nombre de fortunes dépassant les cinq millions : enrichissement spectaculaire » (Mitterand, 2008, p. 61). Cette gentry de l'époque, pour qui « les marques de la richesse prennent plus de valeur que sa possession " (Tulard, 1995, p. 1343), érige le paraître en une religion nouvelle, en fait la quintessence de sa vie. L'exemple vient des Tuileries : Napoléon III et Eugénie soutiennent l'industrie du luxe et le commerce parisien, jouant ainsi un rôle moteur dans la diffusion de la mode apparue à la cour dans les salons de l'aristocratie et auprès de la bourgeoisie (voir : Yon, 2016, p. 16). Ainsi, présenter une nouvelle toilette, un collier de diamants, des chevaux de race ou un équipage fastueux qu'on vient d'acquérir est un point immuable du comme il faut aristocratique et haut-bourgeois. Les tableaux tels que «le défilé des calèches du Bois de Boulogne, l'éclat des toilettes féminines, [...] la décoration pléthorique des salons et de leur ameublement, les réceptions et les fêtes " (Mitterand, 2008, p. 61) véhiculent le raffinement, l'élégance, mais aussi un certain excès dans l'affichage de la prospérité par les hautes classes de l'époque.

Quelques milieux mondains, composés surtout de personnes jeunes, développent même toute une "culture de la jouissance et du gaspillage, une existence luxueuse sur les boulevards parisiens »(Becker, 2011, p. 39). Ce cercle de dandys et de coquettes, connu sous le nom de "Tout-Paris »,

reste [...] fixé sur l'aspect extérieur et sur les signes matériels qui confirment sa conviction d'appartenir à une élite sociale [...]. [Ces gens] tiennent à la qualité de leur tenue et dispersent leur temps en le consacrant à d'interminables distractions, à savoir les bals, les spectacles, les concerts [...]. Ils se font remarquer par le faste de leurs équipages, par une élégance hors du commun, ainsi que des maisons où règne le luxe. (Kociubińska, 2015, p. 30-31)

Aussi bien en tant que romancier qu'en tant que chroniqueur, Zola " peint les lieux où la société riche et élégante étale son luxe et s'adonne au plaisir " (Tulard, 1995, p. 1343). La scène la plus propice à l'affichage de la prospérité est sans doute le Bois de Boulogne récemment aménagé, lieu de promenade favori des riches et repère incontournable de quiconque se veut mondain. Effectivement, la promenade en calèche ou à cheval dans ce parc énorme, appelé familièrement « le Bois » tout court, est devenue un des rites quotidiens de l'aristocratie et de la haute bourgeoisie : qu'il fasse beau ou qu'il pleuve à torrents, qu'on prenne un plaisir à s'y rendre ou qu'on s'y ennuie à mourir, personne ne se dérobe à l'obligation d'y aller. Car, contrairement aux salons mondains, où il sied d'être invité pour pouvoir s'y présenter, le Bois, ce « grand salon de Paris [...], offre cette liberté de rencontrer des personnes influentes, de s'y faire remarquer et d'avancer dans la vie» (Benhamou, 2008, p. 2-3), ce qui en fait un lieu d'importance capitale. 
Le Bois de Boulogne, un des derniers vestiges de l'enceinte forestière entourant Paris au Moyen Âge, avait été, en 1815 encore, une forêt éloignée du centre de la capitale où campaient les armées alliées contre Napoléon I ${ }^{\text {er }}$. Sous le Second Empire, il a subi une métamorphose complète qui a transformé le lieu, réputé sauvage et dangereux, en promenade publique dont les Parisiens étaient très fiers. Les chiffres cités par le Dictionnaire du Second Empire confirment l'échelle impressionnante des travaux effectués : la superficie du Bois fut portée à presque 850 hectares ; on a empierré plus de 58 kilomètres de routes carrossables et ensablé presque 12 kilomètres d'allées cavalières ; plus de 26 kilomètres de sentiers forestiers ont été rendus utilisables pour les promeneurs (voir : Tulard, 1995, p. 670). La création de deux lacs artificiels séparés par une cascade, de fausses grottes, la plantation de plus de 400000 arbres ont complété la transformation de cet ancien domaine de chasse en un immense parc à l'anglaise, un " Hyde Park » parisien, lié à la ville par la large avenue de l'Impératrice, menant à la place de l'Étoile, et par les pistes cavalières allant jusqu'à la place de la Concorde. D'autres attractions encore ont vu le jour, accessibles à tous grâce au développement du réseau de transport : "Un hippodrome et un jardin d'acclimatation furent aménagés à Longchamp ; le chemin de fer de ceinture puis un service de bateaux permirent à ceux qui n'avaient pas de voiture de se rendre au Bois» (Tulard, 1995, p. 968). On a fait tout pour que l'endroit « adapte ses lignes artistiques aux exigences du grand nombre et au bien-être des promeneurs » (Tulard, 1995, p. 670):

[...] [L'architecte] Davioud [...] y composa les vingt-quatre pavillons et chalets construits pour l'habitation des gardes, les kiosques, les embardacères, les grottes, les balustrades en ciment qui imitaient le bois, les abris cavaliers, les exèdres, les différents restaurants. Il dessina le mobilier urbain : poteaux indicateurs, porte-affiches, bancs de repos, grilles d'entrée. (De Moncan, 2012, p.110-112)

L'effet de cette entreprise gigantesque est une grande réussite : même les sceptiques avouent que le Bois est devenu " la vraie promenade de l'Europe " (Tulard, 1995, p. 671). Les ennemis déclarés du régime, comme la comtesse Marie d’Agoult - essayiste, journaliste et critique d'art, se montrent aussi admiratifs devant le tableau que le nouveau Bois présente désormais aux yeux des promeneurs : « [d]es gondoles sur les lacs, des cafés dans les chalets, des courses sur le turf, des revues sur les pelouses, des jeux, des concerts » qui attirent chaque jour « quatre ou cinq rangs d'équipages ", avec " les dames du "high life", comme on les appelle, et les demoiselles, les galantes avec qui il leur plaît de se confondre ", accompagnées des cavaliers " rapides, le cigare en bouche» (De Moncan, 2012, p. 114).

L'image des rangs de véhicules inondant les allées du Bois à des heures fixes revient de façon récurrente chez divers auteurs décrivant ce parc, témoignant de l'importance des voitures en tant que manifestations les plus visibles du niveau de vie de la haute société. On se souvient, à ce propos, du premier chapitre de La Curée 
où la calèche de Renée Saccard est bloquée, au retour du Bois, par l’encombrement de plusieurs dizaines de carrosses dont le faste et l'élégance contribuent au pittoresque de la scène:

Au milieu des taches unies, de teinte sombre, que faisait la longue file des coupés [...], brillaient le coin d'une glace, le mors d'un cheval, la poignée argentée d'une lanterne, les galons d'un laquais haut placé sur son siège. Ça et là, dans un landau découvert, éclatait un bout d'étoffe, un bout de toilette de femme, soie ou velours. [...] Ce pétillement des harnais et des roues, ce flamboiement des panneaux vernis dans lesquels brûlait la braise rouge du soleil couchant, ces notes vives que jetaient les livrées éclatantes perchées en plein ciel et les toilettes riches débordant les portières, se trouvèrent ainsi emportés par le trot des attelages. (Zola, 1978, p. 10)

En effet, la voiture particulière avec toutes ses variantes (break, calèche, landau, etc.), ce mode de déplacement tout récent dû au nouveau réseau de liens spatiaux mis en place par le développement de la voirie dans Paris, aussi bien intra qu'extra muros, est un must-have de tout représentant des classes riches. L'essor de l'équipage entraîne d'ailleurs le développement considérable de la branche d'artisanat correspondante, ce qui fait vite de Paris la capitale européenne de la carrosserie de luxe (voir : Libourel et Florençon, 2012, p. 25).

Ce moyen glamoureux de transport apparait aussi dans les chroniques zoliennes. Dans celle qui s'intitule Une promenade de Thiers dans les rues de Paris («La Cloche" du 9 octobre 1872), le chroniqueur, en observant la voiture de Thiers, se souvient d'une promenade de Napoléon III dans le Bois ; ce texte pourrait être considéré comme une esquisse du fragment du roman cité ci-dessus : le même spectacle du soleil couchant observé du même endroit va de pair avec la même somptuosité du magnifique attelage et avec toute la symbolique de richesse et de satiété qu'il véhicule :

[...] dans la poussière d'or du soleil couchant, l'empereur passait. Il y avait, en avant, quatre piqueurs vêtus de vert, galonnés d'or, qui couraient sur leurs grands chevaux, qui faisaient faire place. Puis le landau impérial apparaissait, dans le vide qu' ils laissent derrière eux. L'empereur, à demi couché, blême, saluait. Et la foule, charmée de l'attelage, heureuse de la belle allure des piqueurs, s'enthousiasmait, agitait des chapeaux, pendant que des messieurs à grosses bottes surveillaient toutes ces mains tendues. C'était le million qui passait, la digestion heureuse, le coffre-fort rempli, toutes les joies satisfaites. (Zola, 1970, p. 181)

C'est en voiture aussi que se rend au Bois la baronne Georgette, personnage principal d'une "chronique déguisée en nouvelle (Mitterand, 2013, p. 65) intitulée simplement La semaine d'une Parisienne $e^{2}$, écrite pour le public russe de la revue

2. Zola a collaboré à la revue en question de 1875 à 1880. Le texte y parut le 24 avril 1875, sous le titre « Paris en avril» (Mitterand, 2013, p. 65). 
saint-pétersbourgeoise «Le Messager de l'Europe ». La structure de ce texte, divisée en sept chapitres, suit l'ordre des sept jours de la semaine dont chacun est consacré à une activité mondaine qui, pendant une journée entière, préoccupe particulièrement la protagoniste. Zola ne lui donne pas de patronyme, faisant d'elle un personnage-type, ce qui, selon Henri Mitterand, est conforme aux règles génériques de la nouvelle qui « conserve beaucoup de points communs avec la chronique, brossant des types à grands traits, à la manière des journaux de mode et selon les codes de la frivolité » (Mitterand, 2013, p. 67). Georgette est une jeune veuve de vingt-huit ans, jolie et riche, profitant pleinement de sa liberté et de sa fortune, arrangeant son temps à son gré et attribuant beaucoup d'importance à son image, comme il convient à une "femme charmante [...] qui fréquente le monde" (Zola, 2013, p. 25). Son emploi du temps contient des positions constantes et d'autres variantes; la promenade au Bois fait sans doute partie au premier groupe, étant une "marque de l'oisiveté et thérapeutique de l'ennui » (Mitterand, 2013, p. 67) ; or, ce lundi d'avril, elle prend un aspect tout à fait particulier, presque magique : c'est le premier jour ensoleillé depuis des semaines de froid et de pluie. Georgette ordonne donc d'atteler pour trois heures et va au Bois où, par un temps pareil,

[...] les voitures sont si nombreuses que les chevaux ont dû bientôt se mettre au pas. La masse des équipages, dont les roues sont comme accrochées les unes aux autres, roule lentement autour du lac avec le grondement sourd et ininterrompu d'un torrent. Le doux mouvement de la voiture berce la baronne, et sur ses lèvres erre un sourire vague de plaisir. (Zola, 2013, p. 26)

Toute la société élégante est déjà sur place :

Dans les allées latérales, des cavaliers la saluent. Tout Paris est là. Des amazones passent, leurs longs voiles au vent. Beaucoup de chevaux magnifiques, de beaux équipages. C’est un convoi solennel, une exposition d'un luxe éblouissant. (Zola, 2013, p. 27)

Pour Georgette, qui ne s'intéresse point à la politique mais qui entend parfois dans des salons en parler, cette " exposition d'un luxe éblouissant ", cette parade du beau monde est la meilleure preuve de la légitimité du régime et du bien-être de la nation : «La baronne [...] fait réflexion que les choses doivent aller mieux qu'on ne dit, sans quoi on ne verrait pas tant de gens qui vivent apparemment si bien " (Zola, 2013, p. 27). C'est d'ailleurs la seule observation que la vue de ce « convoi solennel » inspire à la jeune femme qui «n'aime pas les réflexions sérieuses » et qui croit que « [l] e reste ne la concerne pas» (Zola, 2013, p. 27) : son seul souci porte sur le lieu où elle passera ses vacances et son trousseau de robes à y emporter.

C'est avec beaucoup de mépris que Zola fait le portrait des semblables de sa baronne dans une autre chronique, intitulée Les plus beaux jours ( « La Cloche » du 29 octobre 1871). Impitoyable à l'égard des «pantins » mondains qu'il considère comme 
prétentieux, oisifs et vides de toute pensée sérieuse, il n’hésite pas à qualifier le Bois et ses habitués de « bazar mondain » :

[...] dans les allées du Bois, les voitures reparaissent, ces revenantes du temps jadis, avec leurs mêmes attelages superbes [...] Autour du lac, il y a la marquise, l'amie intime des Tuileries, et la duchesse, si aimable pour les jeunes gens, et la baronne, et les simples millionnaires. Tout ce monde sort de sa boîte, sans que les toilettes soient fripées, sans qu'une seule mèche blanche se soit glissée sous les nattes fausses. Les dames sont douillettement couchées au fond des calèches, tandis que les cavaliers passent en les saluant [...] Tout ce monde ne pense à rien, vient là pour s'étaler, et va rentrer pour changer de toilette. C'est le bazar mondain, avec des femmes et des chevaux à vendre [...]. (Zola, 1969, p. 705)

Dans ce texte, la lame de la raillerie sous-jacente est beaucoup plus pointue que dans La semaine d'une Parisienne dont la protagoniste semble inspirer au narrateur, à part une ironie adoucie par le ton léger et frivole du texte, une quasi-indulgence amusée, " comme si [le nouvelliste] avait cédé lui-même à la séduction de son personnage, si charmeur et libre [...]" (Mitterand, 2013, p. 70). Dans Les plus beaux jours, le narrateur pointe du doigt des habitués de la cour qu'il méprise pour leur servilité à l'égard du régime détesté (la marquise), des dames à la morale douteuse (la duchesse), ainsi que des parvenus qui ont fait fortune grâce au Second Empire et qu'il qualifie ironiquement de "simples » millionnaires. La dernière phrase du fragment cité est capitale : mettre dans la même ligne, et même dans la même formule, les femmes et les chevaux, considérés comme des objets "à vendre ", en dit long sur l'opinion du chroniqueur sur la jet-set de l'époque.

À côté des équipages et des attelages, un autre aspect de ce tableau vivant attire l'attention du narrateur et du lecteur : il s'agit de l'importance du code vestimentaire dans l'étalage du luxe des hautes classes. Effectivement, les exigences de la toilette des femmes et des hommes de la meilleure société sont nombreuses et méticuleuses : on s'habille avec soin pour aller au Bois, puis on " rentre pour changer de toilette " (Zola, 1969, p. 705), ce qu'on va effectuer avec autant d'attention, et ce rite est répété plusieurs fois par jour. Selon le Dictionnaire du Second Empire, « [...] la femme élégante a besoin d'une robe pour diverses circonstances dans la même journée : réunions d'après-midi, casino, dîner, grand bal [...]» (Tulard, 1995, p. 838). Il semble que chacun de ces messieurs et dames, surtout les jeunes, ont fait sienne la conception baudelairienne de la mode qui, selon l'auteur des Fleurs $d u$ mal, est une expression de la recherche, inhérente à tout être humain, d'une beauté idéale permettant d'oublier les turpitudes du quotidien, « un symptôme du goût idéal surnageant dans le cerveau humain au-dessus de tout ce que la vie naturelle y accumule de grossier, de terrestre et d'immonde » (Baudelaire, 1956, p. 912). On n'hésite pas à consacrer à cette recherche une part considérable de sa fortune, étant donné qu' « [u]ne robe de couture coûte environ 500 francs en 1855 mais 2000 francs dix ans plus tard » (Tulard, 1995, p. 838). Voilà comment, en 1878, donc à une décennie de distance par 
rapport à l'époque dont il parle, Zola décrit cette génération constamment préoccupée de sa tenue :

La jeunesse de cette époque se distinguait par son élégance, soignait son visage et ses mains avec une coquetterie raffinée, plaçait la question du tailleur au-dessus de toutes les autres, ne paraissant pas dans le monde sans la fleur à la boutonnière. Et [...] l'on croyait profondément à toute cette élégance ; tous croyaient que l'Empire existerait éternellement, tous savaient que l'empereur et l'impératrice aimaient les hommes beaux qui prennent soin d'eux. (Zola, 1970, p. 319)

Zola-chroniqueur de 1878 parle donc d'une même voix que Zola-romancier qui, dix ans plus tôt (1869), en rendant à l'éditeur Lacroix le plan de la série des RougonMacquart, avait indiqué, comme un des sujets principaux de La Curée, " la vie sotte et élégamment crapuleuse de notre jeunesse dorée » (Lanoux, 1978, p. III-IV). Ce qui remplit la tête de la baronne Georgette, lors de la promenade au Bois, en est un bon exemple : d'abord, elle se demande combien de robes il lui faudra emporter en vacances à Biarritz; ensuite, elle est « reprise d'inquiétude pour sa robe. Si elle changeait le velours noir pour de l'atlas noir?»(Zola, 2013, p. 28), inquiétude qui la mène chez sa couturière où elle passe deux heures à discuter du problème des tissus et de la garniture, pour finalement garder le projet initial. Et elle se dépêche de rentrer pour changer de robe car elle dîne ce soir chez sa marraine.

Plusieurs autres chroniques zoliennes prennent pour sujet le luxe des toilettes - surtout celles de femmes - arborées dans les lieux à la mode. Ainsi, Les épaules de la marquise ( L La Cloche » du 21 février 1870) met en scène une femme riche, élégante et raffinée dont l'occupation principale est de «f[aire] son métier, montre[r] ses épaules et gagne[r] sa réputation de jolie femme " (Zola, 1969, p. 263). Les robes courtes («L'Événement illustré » du 16 mai 1868) critique l'impudence des femmes dont les décolletés descendent de plus en plus bas, et se plaint du manque d'originalité dans les tenues des Parisiennes. Dans Lettres de Paris ( Le Messager de l'Europe », juillet 1875), il analyse minutieusement les coupes, les couleurs et les détails décoratifs des habits féminins. L'attention que Zola consacre aux vêtements féminins et qui aboutira, dans La Curée, à l'inégalable perfection descriptive des longs passages consacrés aux robes et aux parures de Renée Saccard, et ensuite à la " folie du chiffon » d'Au Bonheur des Dames, reste d'ailleurs conforme à l'esprit de l'époque : "Sous le règne de Napoléon III, alors que la cour de France est la plus brillante d'Europe, la parure féminine fait figure de métaphore de la société » (Gural-Migdal, 2013, p. 1213). Et cette société, selon le chroniqueur, c'est celle du Paris animé par le carnaval permanent des nouveaux-riches, celle des fortunes dépensées chez les grands couturiers en tenues portées pendant une seule soirée ; celle des bals pendant lesquels les corps de femmes se dénudent de plus en plus sous la couverture ajourée des bijoux ; celle où règnent l'ostentation, le luxe criard et les apparences dont le spectacle est donné quotidiennement au Bois de Boulogne. 
Sous la plume de Zola-chroniqueur, le Paris haut de gamme s'avère donc avant tout une société dont le principal défaut est la vanité, entendue comme un besoin quasi-organique de paraître, d'attirer l'attention, d'être admiré et envié. Si une telle image peut sembler exagérée, il faut pourtant comprendre que, en tant que portraitiste des élites de l'Empire, Zola ne se met plus en position d'un simple « flâneur » ou « enquêteur ", comme il le fait pour ses textes, aussi bien chroniques que romans, dont la mission est de remuer les consciences ; ici, au contraire, sachant que « le journaliste est, par métier, un spécialiste de la parabole sous toutes les formes " (SaminadayarPerrin, 2013, p. 13), il cherche des situations qui pourraient lui servir d'allégories, de généralisations susceptibles de véhiculer la vérité sur la déchéance qui préside, selon lui, à l' " orgie d'appétits et d'instincts » (Lumbroso et Mitterand, 2002, p. 273) qui anime les classes aisées du Second Empire. Les chroniques zoliennes consacrées à ces dernières sont ainsi comme théâtralisées, mettant en scène des personnages-types et des situations dont certains détails sont délibérément exagérés. Ces textes fonctionnent en effet comme des paraboles : " les plaisirs frelatés de la Fête impériale, les tableaux contrastés des boulevards, sujets de prédilection pour la chronique parisienne, ne sont en réalité [...] qu'une vaste mascarade soutenue par les formes les plus inventives du capitalisme » (Saminadayar-Perrin, 2013, p. 12). Autrement dit, le portrait en apparence objectif du Paris des élites est en réalité l'image d'un "Second Empire déliquescent, offrant l'image du luxe et de la corruption " (Sabatier, 2013, p. 103), un prétexte de plus permettant à Zola de diriger l'attention du lecteur vers ce que " la ville-lumière relègue dans ses marges ou ses coulisses " (SaminadayarPerrin, 2013, p. 14), ce qui, à la longue, va contribuer à la légende noire du régime.

Le plus grand jardin public de Paris, espace de distraction et d'oisiveté des nantis des quartiers de l'Ouest, le Bois de Boulogne reste, sous le Second Empire, un «lieu emblématique de la vie parisienne [...] »(Benhamou, 2008, p. 1). Temple du paraître où l'on s'exhibe, on se donne en spectacle et où se tient chaque jour la parade des vanités, l'endroit acquiert le statut du «théâtre du monde, miroir d'une époque " (Benhamou, 2008, p. 10) qui reflète la belle société de son temps, une "société du signe, de l'illusion, qui [...] ne pense qu'au plaisir et au profit » (Tulard, 1995, p. 1343), un monde qui s'achemine inéluctablement vers la décadence.

\section{RÉFÉRENCES}

Baudelaire, Ch. (1956). CEuvres complètes. T. II. Paris, France : Gallimard.

Becker, C., Gourdin-Servenière, G. et Lavielle, V. (1993). Dictionnaire d'Émile Zola. Sa vie, son œuvre, son époque. Paris, France : Laffont.

Becker, K. (2011). Le dandysme littéraire en France au XIX'e siècle. Orléans, France : Éditions Paradigme.

Benhamou, N. (2008). La promenade au Bois dans le roman du XIXe siècle. Récupéré de http:// etudes-romantiques.ish-lyon.cnrs.fr/wa_files/Benhamou.pdf 
Cancellieri, F. (2013). La représentation de Paris dans la littérature du XIXe siècle : entre mythe et réalité. Récupéré de https://portal.education.lu/inno/PROJETS/Projets-D\%C3\%A9tail/ ArtMID/3328/ArticleID/7242/La-repr233sentation-de-Paris-dans-la-litt233rature-duXIXe-si232cle-entre-mythe-et-r233alit233

Dezalay, A. (1967). Cent ans après. Un journaliste bien parisien : Émile Zola portraitiste. Les Cahiers Naturalistes, 34, 114-123.

Gural-Migdal, A. (2003). Introduction. Dans A. Gural-Migdal (dir.), L'écriture du féminin chez Zola et dans la fiction naturaliste (p. 1-26). Berne, Suisse : Peter Lang.

Kociubińska, E. (2015). L'insoutenable pesanteur de l'être. Le dandysme en France au XIX siècle et son rayonnement en Europe. Lublin, Pologne : Wydawnictwo KUL.

Lanoux A. (1978). Préface à La Curée. Dans É. Zola, La Curée (p. III-VIII). Paris, France : Fasquelle.

Libourel, J.-L. et Florençon, P. (dir.). (2012). Voyages et déplacements au XIX siècle, publication des Archives de France / Centre du Microfilm et de la Numérisation. Récupéré de http://projets-educatifs.chateaudespeyran.fr/wp-content/uploads/2012/01/Voyages-etdeplacements-BR.pdf

Lumbroso, O. et Mitterand, H. (éd.). (2002). Les manuscrits et les dessins de Zola. Vol. II : Les racines d'une ouvre. Paris, France : Textuel.

Mitterand, H. (2008). Le Paris de Zola. Paris, France : Hazan.

Mitterand, H. (2013). Notice à La semaine d'une Parisienne. Dans É. Zola, Nouvelles roses. Paris, France : Librairie Générale Française.

Moncan, P. de. (2012). Le Paris d'Haussmann. Paris, France : Les Éditions du Mécène.

Sabatier, C. (2013). La tentation pamphlétaire dans les chroniques de Zola (1866-1872) : pose esthétique ou posture éthique ? Les Cahiers Naturalistes, 87, 103-118.

Saminadayar-Perrin, C. (2013). Zola journaliste : histoire, politique, fiction. Les Cahiers Naturalistes, 87, 5-28.

Tulard, J. (dir.). (1995). Dictionnaire du Second Empire. Paris, France : Fayard.

Yon, J.-C. (2016). Les dessous de la fête impériale. Dossier de l'art, 243, 12-17.

Zola, É. (1969). Euvres complètes. Vol. XIII : Chroniques et Polémiques I. Paris, France : Claude Tchou.

Zola, É. (1970). Euvres complètes. Vol. XIV : Chroniques et Polémiques II. Paris, France : Claude Tchou.

Zola, É. (1978). La Curée. Paris, France : Fasquelle.

Zola, É. (2013). Nouvelles roses. Paris, France : Librairie Générale Française.

RÉSUMÉ : L'œuvre journalistique d'Émile Zola touche tous les aspects de la réalité parisienne de son époque : des sujets « sérieux ", sociaux et politiques, à ceux qu'on peut qualifier de légers, voire frivoles. L'existence de la gentry de la capitale sous le règne de Napoléon III compte, selon l'écrivain, selon ces derniers, étant focalisée sur les apparences et sur un affichage ostentatoire de la richesse. En effet, la vanité s'avère être le défaut principal de la belle société parisienne du Second Empire, et le paraître devient sa nouvelle religion. La scène préférée de la parade quotidienne des mondains est le Bois de Boulogne, récemment transformé en un parc à l'anglaise. L'article 
se penche sur quelques éléments de cette " parade des vanités » dans le contexte de l'attitude hostile de Zola à l'égard du régime qu'il déteste.

Mots-clés : vanité, Bois de Boulogne, Zola, chronique

The theatre of socialites' vanity: the Bois de Boulogne in Emile Zola's chronicles ABSTRACT: Emile Zola's journalistic work deals with every aspect of Parisian reality of his time, from "serious" political and social subjects to slight or frivolous ones. The existence of the Parisian upper classes under the reign of Napoleon III is considered by the author as trivial, based on appearances and an ostentatious display of one's richness. Actually, vanity is the main default of the Parisian high society of the Second Empire, and showing off is their new religion. The everyday parade of wealthy and fashionable people takes place at the Bois of Boulogne, freshly reshaped into an English landscape park. The paper analyzes some elements of this "vanity parade" in relation to Zola's hostile attitude towards the Second Empire.

Keywords: vanity, Bois de Boulogne, Zola, chronicle 